\title{
Glutamate Receptor-Mediated Neurotoxicity in a Model of Ethanol Dependence and Withdrawal in Rat Organotypic Hippocampal Slice Cultures
}

\author{
Elisabetta Gerace ${ }^{1,2 *}$, Elisa Landucci', Daniele Bani ${ }^{3}$, Flavio Moroni², Guido Mannaioni ${ }^{2}$ \\ and Domenico E. Pellegrini-Giampietro ${ }^{1}$ \\ ${ }^{1}$ Section of Clinical Pharmacology and Oncology, Department of Health Sciences, University of Florence, Florence, Italy, \\ 2 Section of Pharmacology and Toxicology, Department of Neuroscience, Psychology, Drug Research and Child Health \\ (NeuroFarBa), University of Florence, Florence, Italy, ${ }^{3}$ Research Unit of Histology and Embryology, Section of Anatomy \\ and Histology, Department of Experimental and Clinical Medicine, University of Florence, Florence, Italy
}

OPEN ACCESS

Edited by:

Tai Wai Yew,

The Chinese University of Hong Kong,

China

Reviewed by:

Euan Robert Brown,

Heriot-Watt University,

United Kingdom

Elena Marcello,

University of Milan, Italy

${ }^{*}$ Correspondence:

Elisabetta Gerace

elisabetta.gerace@unifi.it

Specialty section:

This article was submitted to

Neuropharmacology,

a section of the journal

Frontiers in Neuroscience

Received: 17 September 2018 Accepted: 27 December 2018 Published: 24 January 2019

Citation:

Gerace E, Landucci E, Bani D,

Moroni F, Mannaioni G and Pellegrini-Giampietro DE (2019)

Glutamate Receptor-Mediated Neurotoxicity in a Model of Ethanol Dependence and Withdrawal in Rat Organotypic Hippocampal Slice

Cultures. Front. Neurosci. 12:1053.

doi: 10.3389/fnins.2018.01053
Long-term alcohol use can lead to alterations in brain structure and functions and, in some cases, to neurodegeneration. Several mechanisms have been proposed to explain ethanol (EtOH)-related brain injury. One of the most relevant mechanisms of alcohol-induced neurodegeneration involves glutamatergic transmission, but their exact role is not yet fully understood. We investigated the neurochemical mechanisms underlying the toxicity induced by EtOH dependence and/or withdrawal by exposing rat organotypic hippocampal slices to EtOH (100-300 mM) for 7 days and then incubating the slices in $\mathrm{EtOH}$-free medium for the subsequent $24 \mathrm{~h}$. EtOH withdrawal led to a dose-dependent CA1 pyramidal cell injury, as detected with propidium iodide fluorescence. Electron microscopy of hippocampal slices revealed that not only $\mathrm{EtOH}$ withdrawal but also 7 days chronic EtOH exposure elicited signs of apoptotic cell death in CA1 pyramidal cells. These data were supported by electrophysiological recordings of spontaneus Excitatory Post Synaptic Currents (sEPSCs) from CA1 pyramidal cells. The average amplitude of sEPSCs in slices treated with EtOH for 7 days was significantly increased, and even more so during the first $30 \mathrm{~min}$ of EtOH withdrawal, suggesting that the initial phase of the neurodegenerative process could be due to an excitotoxic mechanism. We then analyzed the expression levels of presynaptic (vGlut1, vGlut2, CB1 receptor, synaptophysin) and postsynaptic (PSD95, GluN1, GluN2A, GluN2B, GluA1, GluA2, mGluR1 and mGluR5) proteins after 7 days $\mathrm{EtOH}$ incubation or after $\mathrm{EtOH}$ withdrawal. We found that only GluA1 and mGluR5 expression levels were significantly increased after EtOH withdrawal and, in neuroprotection experiments, we observed that AMPA and mGluR5 antagonists attenuated $\mathrm{EtOH}$ withdrawal-induced toxicity. These data suggest that chronic EtOH treatment promotes abnormal synaptic transmission that may lead to CA1 pyramidal cell death after EtOH withdrawal through glutamate receptors and increased excitotoxicity.

Keywords: ethanol withdrawal, glutamate receptors, organotypic hippocampal slices, CA1 injury, neuroprotection 


\section{INTRODUCTION}

Alcohol addiction is a chronic, relapsing brain disease characterized by mental and physical health problems with devastating consequences. The World Health Organization (WHO) estimates that there are about 208 million people with alcoholism worldwide (4.1\% of the population over 15 years of age), about 2.3 million people die for a cause alcohol-related and 76.3 million have alcohol use disorders, with social and economic cost, ranging from 1 to $6 \%$ of the Gross Domestic Product (GDP) of a country (World Health Organization [WHO], 2014).

Chronic alcohol exposure and alcohol protracted withdrawal causes profound neuroadaptive changes in neuronal excitability and synaptic plasticity that are the consequences of a complex interplay of biological vulnerability, environmental exposure, and developmental factors (Koob and Le Moal, 2008). The efficacies of current pharmacotherapies targeting alcohol dependence are limited also because alcohol affects diverse neurotransmitter systems in the brain (i.e., dopaminergic, GABAergic, glutamatergic and serotonergic systems) (LeMarquand et al., 1994; Charlet et al., 2013). Importantly, following long-term alcohol consumption, the brain compensates the depressant effects of alcohol to maintain homeostasis between inhibitory and excitatory neurotransmission by increasing excitatory activity and reducing inhibitory activity (Nam et al., 2012; Koob, 2013; Tabakoff and Hoffman, 2013).

Glutamate plays a pivotal role in drug addiction, drug self-administration, reward-related processes and relapse (Kalivas and Volkow, 2005; Gass and Olive, 2008). Increased glutamatergic neurotransmission and hyper-excitability during withdrawal and abstinence are associated with an increased risk for relapse (Tsai and Coyle, 1998), and changes in the glutamatergic system after chronic alcohol intake has been largely described (Nagy et al., 2005; Krupitsky et al., 2007) and numerous reports have demonstrated the functional relationship observed in $\mathrm{EtOH}$ dependent and withdrawn animals and the glutamatergic transmission (Läck et al., 2007; Marty and Spigelman, 2012; Gerace et al., 2016). Elevated levels of glutamate has been measured in the medial prefrontal cortex of alcoholic patients and alcohol-dependent rats during acute withdrawal (Hermann et al., 2012). NMDA receptor alterations and functioning have been described to contribute to neuronal excitation and neurotoxicity during EtOH withdrawal (Butler et al., 2013), whereas AMPA receptors have been shown to play a key role in the regulation of reinforcement and the reward process of EtOH (Stuber et al., 2008; Wang et al., 2012). Preclinical research suggests the important role of metabotropic glutamate subtype 5 (mGlu5) receptor in EtOH drinking (Cozzoli et al., 2009, 2012; Besheer et al., 2010; Sinclair et al., 2012) and in the memory formation responsible for the chronic relapsing nature of alcohol abuse (Obara et al., 2009). Nevertheless, the exact mechanisms by which ethanol exerts its toxic effects through glutamatergic transmission are still unknown.

In the present study, we investigated the neurochemical mechanisms underlying the toxicity induced by $\mathrm{EtOH}$ in organotypic hippocampal slice models of dependence and/or withdrawal. In particular, we investigated the role of ionotropic and metabotropic glutamate receptors in mediating toxicity induced by the chronic exposure to $\mathrm{EtOH}$ and following $\mathrm{EtOH}$ withdrawal.

\section{MATERIALS AND METHODS}

Experiments and animal use procedures were in accordance with the National Institutes of Health Guide for the Care and Use of Laboratory Animals (NIH Publications No. 80-23, revised 1996). The experimental protocols were approved by the Animal Care Committee of the Department of Health Sciences, section of Pharmacology, University of Florence, in compliance with the European Convention for the Protection of Vertebrate Animals used for Experimental and Other Scientific Purposes (ETS no. 123), the Department of Neuroscience, Psychology, Drug Research and Child Health (NeuroFarBa) (ETS no. 176) and the European Communities Council Directive of 24 November 1986 (86/609/EEC). The authors further attest that all efforts were made to minimize the number of animals used and their suffering.

\section{Materials}

Propidium iodide (PI) was purchased from Sigma (St Louis, MO, United States). Tissue culture reagents were obtained from Gibco-BRL (San Giuliano Milanese, MI, Italy) and Sigma (St Louis, MO, United States). 2,3-Dioxo6-nitro-1,2,3,4-tetrahydrobenzo[f]quinoxaline-7-sulfonamide disodium salt (NBQX) and 2-Methyl-6-(phenylethynyl)pyridine (MPEP) hydrochloride were purchased from Tocris (Bristol, United Kingdom).

\section{Preparation of Rat Organotypic Hippocampal Slice Cultures}

Organotypic hippocampal slice cultures were prepared as previously reported Gerace et al., 2012a, 2015, Landucci et al., 2018). Briefly, hippocampi were removed from the brains of 7to 9-day old Wistar rat pups (Harlan, MI, Italy), transverse slices $(420 \mu \mathrm{m})$ were prepared using a McIlwain tissue chopper and then transferred onto $30 \mathrm{~mm}$ diameter semiporous membranes inserts (Millicell-CM PICM03050; Millipore, Italy), which were placed in six well tissue culture plates containing $1.2 \mathrm{ml}$ medium per well. The culture medium consisted of 50\% Eagle's minimal essential medium, 25\% heat-inactivated horse serum, 25\% Hanks' balanced salt solution, $5 \mathrm{mg} / \mathrm{ml}$ glucose, $2 \mathrm{mM}$ L-glutamine, and $3.75 \mathrm{mg} / \mathrm{ml}$ amphotericin B. Slices were maintained at $37^{\circ} \mathrm{C}$ in an incubator in atmosphere of humidified air and $5 \% \mathrm{CO}_{2}$ for 10 days. Before experiments all slices were screened for viability by incubating them for $30 \mathrm{~min}$ with PI $(5 \mu \mathrm{g} / \mathrm{ml})$; slices displaying signs of neurodegeneration were discarded from the study.

\section{Ethanol Exposure and Drug Treatment in Organotypic Hippocampal Slices}

Rat organotypic hippocampal slice cultures were exposed for 7 days to 100,150 , or $300 \mathrm{mM}$ of $\mathrm{EtOH}$ (corresponding 
respectively to $4.6,6.9$, or $13.8 \mathrm{~g} / \mathrm{l}$ ) after 10 days of culture in vitro as described in Gerace et al. (2016). The medium was changed every day adding ethanol to the fresh culture medium. After 7 days of EtOH treatment, some of the slices were incubated in $\mathrm{EtOH}$-free medium or in ethanol-free medium plus the AMPA antagonist NBQX and/or the metabotropic Glu5 antagonist MPEP for $24 \mathrm{~h}$ before they were assessed for neuronal injury using PI fluorescence. As discussed (Gerace et al., 2012b, 2016; Landucci et al., 2016), the concentrations of drugs used in organotypic hippocampal slice experiments are generally somewhat higher than those expected from their $\mathrm{Kd}$ values and those used in cell cultures. This is due to the fact that they diffuse slowly through the thickness of brain tissue in vitro. Moreover, in the case of organotypic slices statically cultured on semiporous membrane inserts at the interface between culture medium and gas atmosphere, the concentrations of drugs need to be further increased because only the bottom of the slice is exposed to the bathing medium to which the drug is added and only a fraction of the drug diffuses across the membrane and reaches the tissue.

\section{Assessment of CA1 Pyramidal Cell Injury}

Propidium iodide $(5 \mu \mathrm{g} / \mathrm{ml})$ was added to the medium either at the end of the 7-day $\mathrm{EtOH}$ incubation period or $24 \mathrm{~h}$ after it was removed from the medium. Thirty minutes later, fluorescence was viewed using an inverted fluorescence microscope (Olympus IX-50; Solent Scientific, Segensworth, United Kingdom) equipped with a xenon-arc lamp, a low-power objective (4X) and a rhodamine filter. Images were digitized using a video image obtained by a CCD camera (Diagnostic Instruments Inc., Sterling Heights, MI, United States) controlled by software (InCyt Im $1^{\mathrm{TM}}$; Intracellular Imaging Inc., Cincinnati, OH, United States) and subsequently analyzed using the Image-Pro Plus morphometric analysis software (Media Cybernetics, Silver Spring, MD, United States). In order to quantify cell death, the CA1 hippocampal subfield was identified and encompassed in a frame using the drawing function in the image software (ImageJ; NIH, Bethesda, MD, United States) and the optical density of PI fluorescence was detected. There was a linear correlation between CA1 PI fluorescence and the number of injured CA1 pyramidal cells as detected by morphological criteria (Pellegrini-Giampietro et al., 1999).

\section{Electron Microscopy in CA1 Region of Hippocampal Slices}

At the end of the experiments the slices were washed with cold $0.01 \mathrm{M}$ phosphate-buffered saline, $\mathrm{pH} 7.4$ and were directly fixed in cold $4 \%$ glutaraldehyde in $0.1 \mathrm{M}$ sodium cacodylate buffer $(\mathrm{pH}$ 7.4) overnight at $4^{\circ} \mathrm{C}$ and post-fixed in cold $1 \%$ osmium tetroxide in $0.1 \mathrm{M}$ phosphate buffer ( $\mathrm{pH} 7.4$ ) for $1 \mathrm{~h}$ at room temperature as described in Gerace et al. (2016). The samples were dehydrated in graded acetone, passed through propylene oxide, and embedded in Epon 812. Ultrathin sections were stained with uranyl acetate and alkaline bismuth subnitrate and examined under a JEM 1010 electron microscope (Jeol, Tokyo, Japan) at $80 \mathrm{kV}$.

\section{Electrophysiological Recordings in Organotypic Hippocampal Slices}

Whole-cell voltage-clamp recordings were performed as described in Gerace et al. (2016). Briefly, inward currents were recorded in CA1 pyramidal cells from organotypic hippocampal slices chronically treated with $150 \mathrm{mM}$ of EtOH or under control conditions. Slices were removed from the culture insert and placed into a recording bath submerged with ice-cold artificial cerebrospinal fluid (ACSF) containing (in mM): EtOH 150, $\mathrm{NaCl}$ 130, $\mathrm{KCl} 3.5, \mathrm{Na}_{2} \mathrm{H}_{2} \mathrm{PO}_{4} 3, \mathrm{NaHCO}_{3}$, glucose, $\mathrm{MgCl}_{2} 1.5$ and $\mathrm{CaCl}_{2} 1.5$ at $\mathrm{pH} 7.4$ and oxygenated with $95 \% \mathrm{O}_{2} / 5 \% \mathrm{CO}_{2}$ ).

Recording microelectrodes were prepared from borosilicate glass (WPI Inc; Sarasota, FL, United States) by a Narishige Instruments micropipette puller (Tujunga, CA, United States) (resistance ranging from 3 to $5 \mathrm{M} \Omega$ ) and filled with internal solution of the following composition (in $\mathrm{mM}$ concentrations): K-gluconate 142.5 , potassium methylsulfate $20, \mathrm{NaCl} 8$, Hepes 10, EGTA 0.1, MgATP 2, and GTP 0.2. The pH of the internal solution was adjusted to 7.2 with $\mathrm{KOH}$ and the osmolarity was adjusted to $300 \mathrm{mOsm}$ with $\mathrm{H}_{2} \mathrm{O}$ and sucrose. After establishing a GIGA seal a whole-cell configuration was achieved by rupturing the membrane. Recordings were done in voltage clamp configuration (holding potentials $-60 \mathrm{mV}$ ) using a Multiclamp preamplifier (Axon Instruments; Foster City, CA, United States) and filtered at $5 \mathrm{kHz}$. All the data were acquired, stored and analyzed on a PC using the pCLAMP (Axon Instruments, Foster City, CA, United States) and GraphPad softwares. Traces were filtered by a digital Gaussian filter (Clampit facility, low pass, $200 \mathrm{~Hz}$ ). The frequency and peak amplitude of detected events were analyzed using Mini Analysis Program (Synaptosoft Inc., Fort Lee, NJ, United States) ${ }^{1}$. The AMPA receptor blocker NBQX $(10 \mu \mathrm{M})$ was added at the end of each experiment to verify that the spontaneous excitatory post synaptic currents were AMPA receptor-mediated.

\section{Western Blot Analysis}

Cultured slices were washed with cold $0.01 \mathrm{M}$ phosphate-buffered saline, $\mathrm{pH} 7.4$ and 8 slices/sample were gently transferred and dissolved in a tube containing $1 \%$ SDS. Total protein levels were quantified using the Pierce (Rockford, IL, United States) BCA (bicinchoninic acid) Protein Assay. Forty $\mu \mathrm{g}$ of proteins were resolved by electrophoresis on a 8 or $12 \%$ SDS-polyacrylamide gel and transferred onto nitrocellulose membranes using the transblot TURBO (Bio-Rad, Hercules, CA, United States). Blots were probed overnight at $4^{\circ} \mathrm{C}$ with the polyclonal rabbit vGluT1, vGluT2, GluN2A, GluA1, metabotropic Glu1, PSD95, polyclonal goat GluA2, GluN1 and monoclonal mouse metabotropic Glu5 antibodies (Millipore, Italy), synaptophysin and GluN2B antibodies (Thermo Scientific, Rockford, IL, United States), C-terminal rabbit anti-CB1 polyclonal antibody (generously provided by Dr. Ken Mackie, University of Washington, Seattle, WA, United States), all diluted 1:1000 (Gerace et al., 2012b, 2016). Immunodetection was performed with secondary antibodies [1:2000 anti-mouse or anti-rabbit IgG from donkey or anti

\footnotetext{
${ }^{1}$ www.synaptosoft.com
} 
goat (Amersham Biosciences, United Kingdom) conjugated to horseradish peroxidase. The reactive bands were detected using chemiluminescence (ECLplus; Euroclone, Padova, Italy). Quantitative analysis was performed using the QuantityOne analysis software (Bio-Rad, Hercules, CA, United States).

\section{Statistical Analysis}

Data are presented as means \pm SEM of $n$ experiments. Statistical significance of differences between PI fluorescence intensities or Western blot optical densities was evaluated by performing oneway ANOVA followed by Dunnet's test for multiple comparisons or by the Kolmogorov-Smirnov test (sEPSC recordings). All statistical calculations were performed using GRAPH-PAD PRISM v. 5 for Windows (GraphPad Software, San Diego, CA, United States). A probability value $(P)$ of $<0.05$ was considered significant.

\section{RESULTS}

Figure 1 shows that organotypic hippocampal slices exposed to chronic EtOH for 7 days and then incubated in EtOH free-medium for the subsequent $24 \mathrm{~h}$ displayed a selective and dose-dependent CA1 pyramidal necrotic cell death. The concentrations of EtOH decreased within $24 \mathrm{~h}$ from $127 \mathrm{mM}$ $(5.8 \mathrm{~g} / \mathrm{l})$ to $32 \mathrm{mM}(1.5 \mathrm{~g} / \mathrm{l})$, which is similar to the blood alcohol concentration detected in the blood of alcoholics in the emergency room as shown in Gerace et al. (2016).

Electron microscopy confirmed what has been observed with PI fluorescence and shows that after $\mathrm{EtOH}$ withdrawal the slices undergo to neuronal death (Figures 2E,F). Moreover, CA1 pyramidal cell bodies exposed to $\mathrm{EtOH}$ for 7 days displayed suffering mithocondria, accumulation of lipofuscins and intercellular empty spaces (Figure 2C,D) as compared to control organotypic slices (Figures 2A,B), suggesting that not only withdrawal but also 7 days chronic EtOH exposure elicited signs of apoptotic cell death in CA1 pyramidal cells.

In order to understand the molecular mechanisms underlying the toxicity induced by $\mathrm{EtOH}$ withdrawal in organotypic hippocampal slices, we performed electrophysiological experiments by using the patch clamp technique. We recorded sEPSCs from CA1 pyramidal cells exposed to $\mathrm{EtOH}$ for 7 days (as shown in Figure 3B) and during the first $30 \mathrm{~min}$ of $\mathrm{EtOH}$ withdrawal. Figure 3A represents an example trace of the effect of the AMPA antagonist NBQX on sEPSCs in control slice, showing that under our experimental conditions sEPSCs are mainly if not exclusively AMPA-mediated. We did not observe differences in the holding current between control slices and slices chronically exposed to $\mathrm{EtOH}$ for 7 days $(100 \pm 44$ pA, $n=5$ vs. $140 \pm 32.6$ $\mathrm{pA}, n=5$, respectively). Figure 3B show examples of sEPSCs recorded under control conditions, after chronic incubation with EtOH for 7-days and after $15 \mathrm{~min}$ of $\mathrm{EtOH}$ withdrawal. Our results show that chronic EtOH exposure increases the amplitude but not the frequency of sEPSCs (Figure 3). Interestingly, EtOH withdrawal further increases sEPSCs amplitude without affecting sEPSCs frequency compared to chronic EtOH exposure, thus indicating that glutamatergic transmission is involved in both $\mathrm{EtOH}$ dependence and withdrawal processes (Figure 3). Kolmogorov-Smirnov analyses confirms that both the chronic incubation with EtOH or its withdrawal produced a rightward shift of the cumulative probability distribution of sEPSC amplitude (Figure 3B, lower panel).

We then performed Western blotting analysis in organotypic hippocampal slices exposed to EtOH using antibodies directed against specific pre- and post-synaptic proteins (Figures 4, 5, respectively). Chronic incubation with $\mathrm{EtOH}$ for 7 days and its removal from the medium did not change the expression of the presynaptic proteins vGluT1, vGluT2, CB1 receptors and synaptophysin (Figure 4). On the other hand, at the postsynaptic level, EtOH withdrawal induced an increased expression of the AMPA subunit GluA1 and of the metabotropic Glu5 receptors, while chronic EtOH exposure showed a trend toward an increased expression without reaching a statistical significance (Figures 5B,F) of the same proteins. No changes for NMDA subunits (GluN1, GluN2A, and GluN2B) nor for the scaffold protein PSD95, GluA2 AMPA subunit and mGluR1 receptors were observed at the postsynaptic level (Figure 5) in both chronic EtOH exposure and $\mathrm{EtOH}$ withdrawal.

These results propose a central role for AMPA and metabotropic Glu5 receptors in EtOH -induced CA1 toxicity and suggest AMPA and mGlu5 as possible therapeutic targets. Therefore, we used a therapeutic approach in vitro by exposing the slices to the AMPA competitive antagonist NBQX and to the selective mGluR5 non-competitive antagonist MPEP during the $24 \mathrm{~h} \mathrm{EtOH}$ withdrawal period. We observed that both these agents were able to significantly attenuate $\mathrm{EtOH}$ withdrawalinduced neurotoxicity in the CA1 hippocampal subregion $(34 \pm 2.7 \%$ and $26.7 \pm 10.7 \%$ NBQX and MPEP, respectively) (Figure 6) confirming that AMPA channels and mGlu5 receptors are implicated in $\mathrm{EtOH}$ withdrawal injury.

\section{DISCUSSION}

In the present study, we investigated the neurochemical mechanisms underlying the toxicity induced by $\mathrm{EtOH}$ in organotypic hippocampal slice models of $\mathrm{EtOH}$ dependence and/or withdrawal. Rat organotypic hippocampal slices (10 DIV) were exposed for 7 days to concentrations of EtOH ranging between 100 and $300 \mathrm{mM}$ (corresponding to 4.6 and $13.8 \mathrm{~g} / \mathrm{l}$ respectively). As reported in Gerace et al. (2016), the actual EtOH concentration measured in our culture media decreased within $24 \mathrm{~h}$ from $127 \mathrm{mM}(5.8 \mathrm{~g} / \mathrm{l})$ to $32 \mathrm{mM}$ (1.5 g/l), which reproduces the blood alcohol concentration oscillations typical of a frequent drinker and is compatible with the high EtOH peak concentrations (between 3 and $4 \mathrm{~g} / \mathrm{l}$ ) detected in the blood of alcoholics in the emergency room. The slices were then incubated in EtOH-free medium for the subsequent $24 \mathrm{~h}$ to mimic EtOH withdrawal. Under these conditions, we observed that EtOH withdrawal led to a dose-dependent CA1 pyramidal necrotic cell death as detected by PI fluorescence and confirmed by electron microscopy, revealing clear signs of injury in CA1 pyramidal cell bodies. Our results are in agreement with previous studies showing neuronal damage and permanent impairment 
A

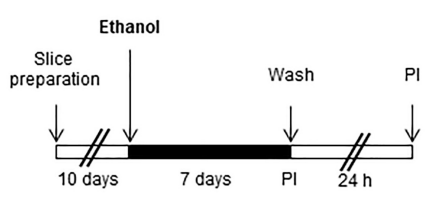

B

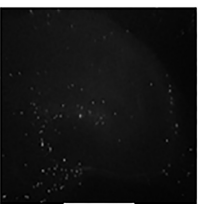

CRL

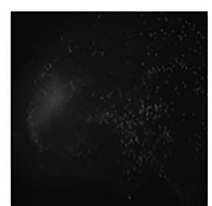

Chronic EtOH

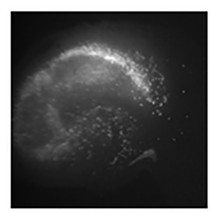

EtOH withdrawal
C

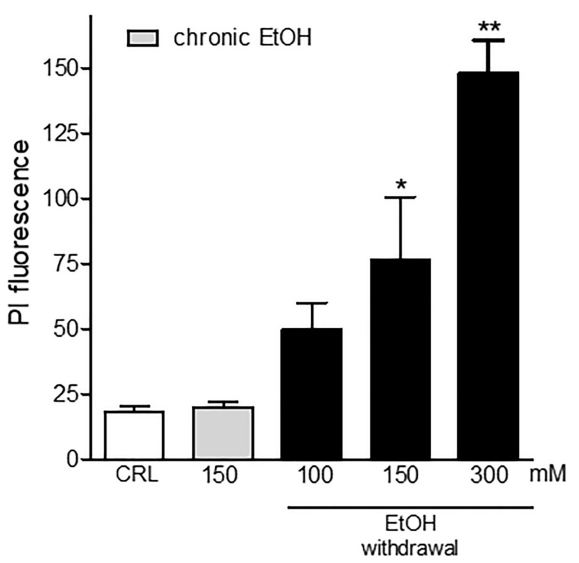

FIGURE 1 | Ethanol withdrawal induces cell death in mature organotypic hippocampal slices. (A) Experimental protocol showing hippocampal slices cultured for 10 days in vitro and exposed to 100, 150, $300 \mathrm{mM}$ of ethanol (corresponding to 4.6, 6.9, or $13.8 \mathrm{~g} / \mathrm{l}$ of plasmatic EtOH concentration in humans) for 7 days. At the end of this period (Chronic ethanol), ethanol was removed from the medium. $24 \mathrm{~h}$ later (Withdrawal) the fluorescent dye propidium iodide (PI) was added to the medium to assess neuronal injury. (B) Mature hippocampal slices, photographed under fluorescence optics, displaying background levels of fluorescence under control or chronic ethanol condition (150 mM) and an intense PI labeling after ethanol withdrawal (150 mM), showing a selective CA1 pyramidal cell injury. (C) Cell injury was assessed using the fluorescent dye propidium iodide at the end of the chronic EtOH treatment (150 mM) and after $24 \mathrm{~h}$ of EtOH withdrawal. Quantitative data are expressed as CA1 PI fluorescence. Values represent the mean \pm SEM of 5 experiments in ethanol withdrawal condition. ${ }^{*} p<0.05$ and $* * p<0.01$ vs. basal PI fluorescence (ANOVA + Dunnet's test).
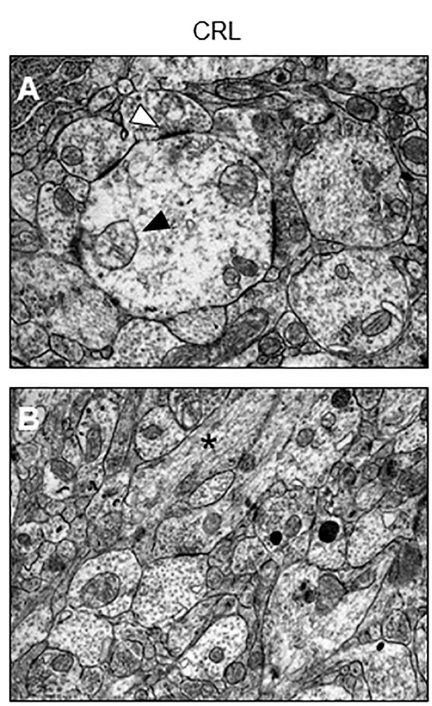
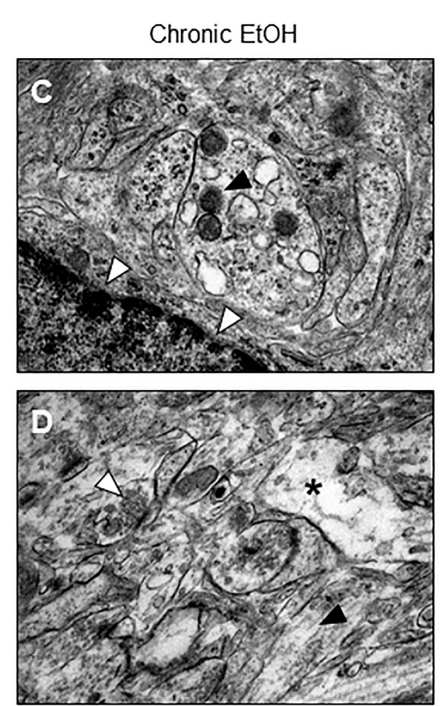

EtOH withdrawal
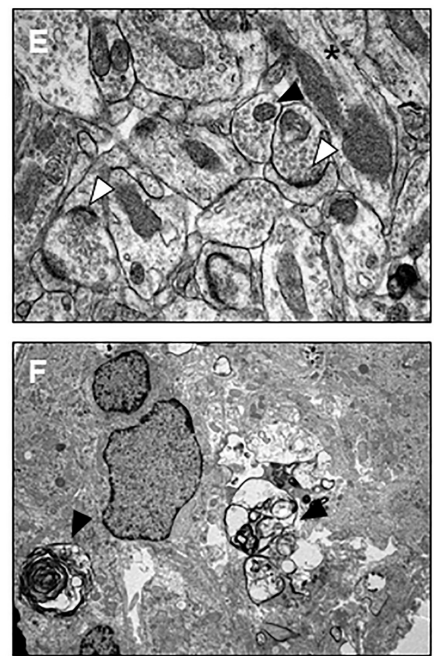

FIGURE 2 | Electron microscopic evidence for apoptotic cell death in organotypic hippocampal slices. (A,B) Control healthy CA1 pyramidal cells showing healthy mithocondria (black arrow) and synapses rich in vescicles (white arrow) (A) and in longitudinally aligned microtubules in the neuronal processes (black asterisk) (B). (C,D) CA1 pyramidal cells from chronic ethanol slices display suffering mithocondria (black arrow), accumulation of lipofuscins (white arrows) (C) and intercellular empty spaces (black asterisk), but regular synapses (white arrow) and in longitudinally aligned microtubules in the neuronal processes (black arrow) (D). (E,F) Electron microscope morphology in control organotypic slices showing normal synapses with presynaptic vescicles (white arrows) and in longitudinally aligned microtubules in the neuronal processes (black asterisk) (E) and clear signs of apoptosis (black arrows) (F).

in synaptic transmission of hippocampal neurons (Prendergast et al., 2000; Harris et al., 2003; Self et al., 2005).

Interestingly, a previous paper from our laboratory shows that, under the same experimental conditions, the deprivation of
EtOH is not toxic in slices cultured for only 2 DIV, suggesting that slices that have not reached complete maturation in vitro may respond to prolonged EtOH incubation in a different manner as compared to more mature slices, resulting in greater resistance 
A

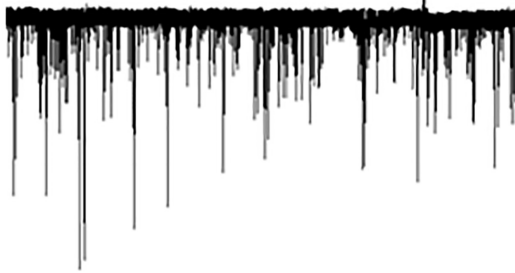

B

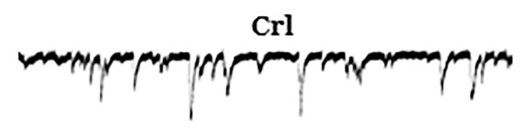

Chronic EtOH

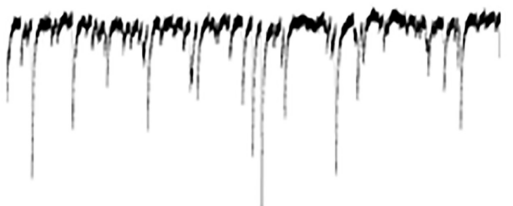

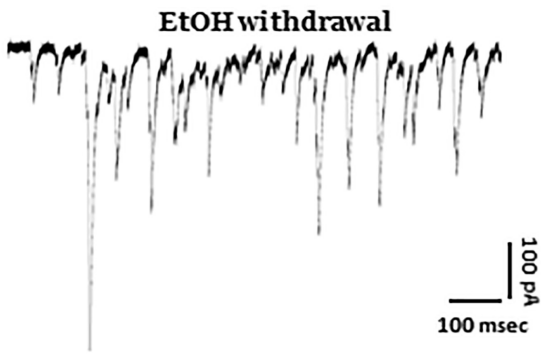

C
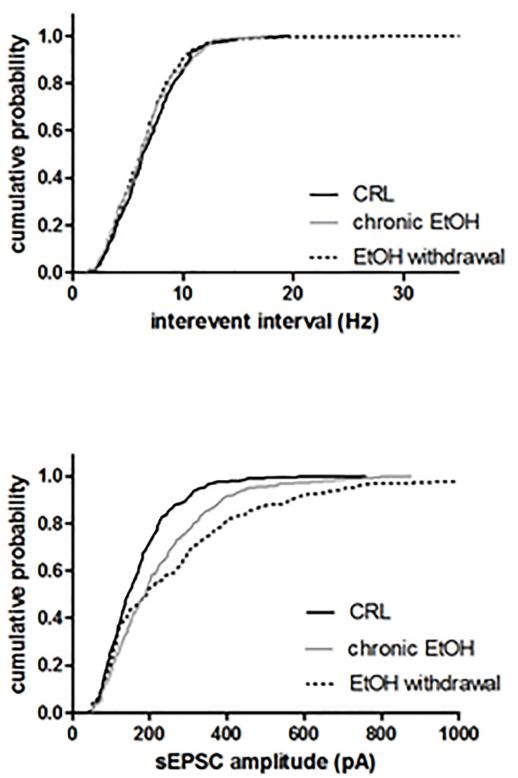

D

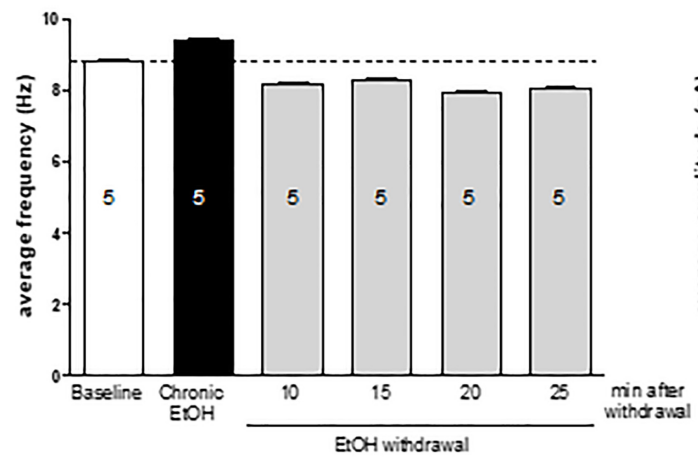

$\mathbf{E}$

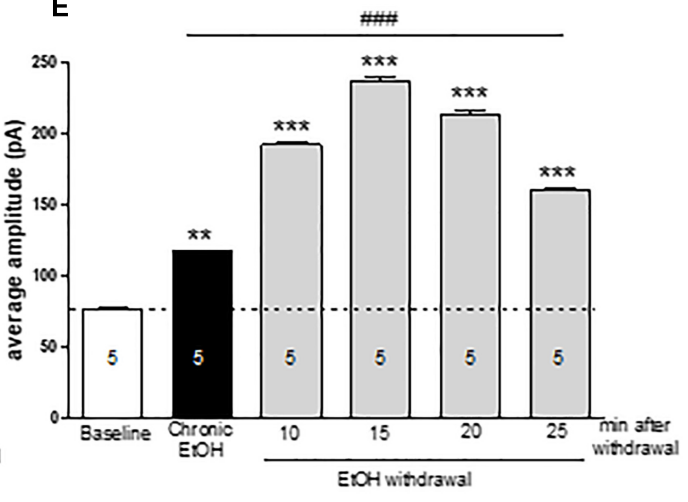

FIGURE 3 | Chronic ethanol and ethanol withdrawal increase the amplitude of sEPSCs in CA1 pyramidal cells of organotypic hippocampal slices. Experiments were conducted as described in Figure 1A. (A) Example trace showing the effect of the AMPA antagonist NBQX on sEPSCs recorded in CA1 pyramidal neurons of control organotypic hippocampal slices in whole-cell voltage clamp configuration. (B) Representative traces showing sEPSCs recorded in CA1 pyramidal neurons of organotypic hippocampal slices in whole-cell voltage clamp configuration under control, chronic ethanol incubation and ethanol withdrawal conditions.

(C) Cumulative probability plots demonstrating the effect of EtOH withdrawal on sEPSC interevent interval and amplitude. EtOH withdrawal caused a significant shift in the amplitude distributions, indicating a significant increase in the amplitude of sEPSCs [Kolmogorov-Smirnov (K-S) statistic CRL vs. chronic EtOH and CRL vs. EtOH withdrawal $p<0.0001]$. On the contrary, the cumulative probability plots on sEPSC interevent interval did not show any significant changing (K-S statistic CRL vs. chronic EtOH $p=0.977, \mathrm{CRL}$ vs. EtOH withdrawal $p=0.462$ ). Bar graphs displaying the values of sEPSC frequency (Hz) (D) and amplitude (pA) (E) during the time course of the last 5 min of chronic ethanol treatment and the first 30 min of ethanol withdrawal. Bars represent the mean of at least 5 experiments. ${ }^{* * *} p<0.001$ vs. CRL and ${ }^{* *} p<0.01,{ }^{*} \# p<0.01$ vs. chronic EtOH (ANOVA + Tukey's $w$ test). The number of cells is indicated on the bar graphs. 
A

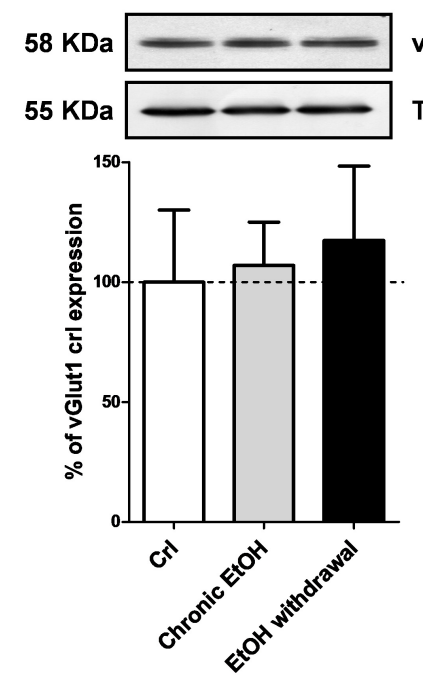

C
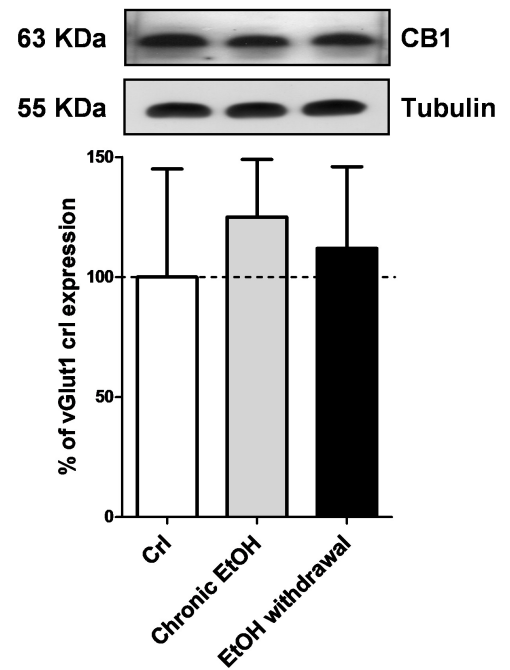

B
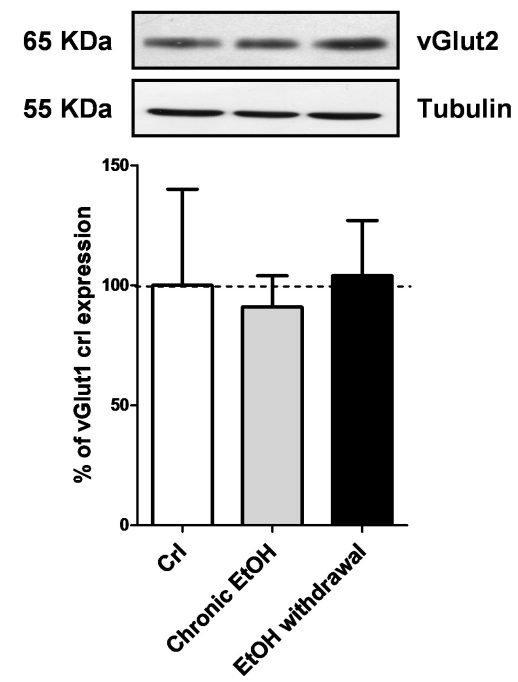

D
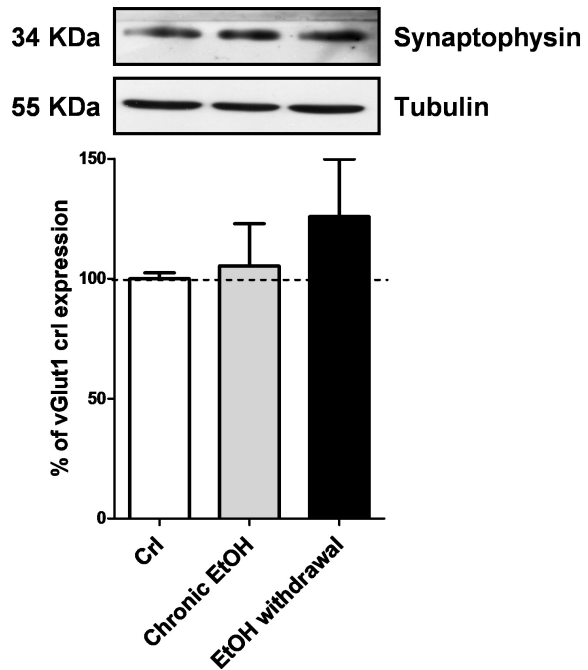

FIGURE 4 | Chronic ethanol and ethanol withdrawal do not modify the expression of presynaptic proteins in organotypic hippocampal slices. Experiments were conducted as described in Figure 1A. At the end of the experiments hippocampal slices were lysed and processed for Western blot. Top: representative Western blots using antibodies against the pre-synaptic proteins vGlut1, vGlut2, CB1 and synaptophysin (A-D). The numbers on the left indicate the position of the molecular mass markers $(\mathrm{kDa})$. Tubulin was used as loading control. Bottom: quantitative analysis of immunoreactive bands, showing that chronic ethanol nor ethanol withdrawal modify the expression of vGlut1, vGlut2, CB1 and synaptophysin. Data are expressed as percentage of control. Bars represent the mean \pm SEM of at least three experiments. ${ }^{*} P<0.05$ vs. CRL (ANOVA + Dunnet's test).

to EtOH toxicity upon its removal (Gerace et al., 2016). The differential age-dependent effects of $\mathrm{EtOH}$ were highlighted also by electron microscopy, which revealed, despite the lack of toxicity observed with PI, a clear disorganization of microtubuli in neural processes after chronic EtOH exposure in immature slices (Gerace et al., 2016). On the contrary, microtubuli alignment was observed in mature slices chronically exposed to $\mathrm{EtOH}$ in the same CA1 neurons, but also ultrastructural signs of neuronal suffering like distressed mitochondria, accumulation of lipofuscins and intercellular empty spaces as compared to control slices. These results suggest that not only withdrawal but also chronic EtOH exposure may elicit signs of apoptotic cell death in CA1 pyramidal cells.

These data are supported by our electrophysiological recordings of AMPA-mediated sEPSCs from CA1 pyramidal cells after 7 days of $\mathrm{EtOH}$ exposure and during the first $30 \mathrm{~min}$ of EtOH removal, which revealed a significant increase in the average amplitude but not in the frequency of sEPSCs that was induced not only by EtOH withdrawal but was also present in slices chronically exposed to $\mathrm{EtOH}$, thus suggesting that 


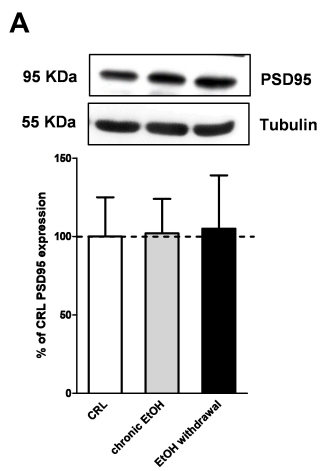

B

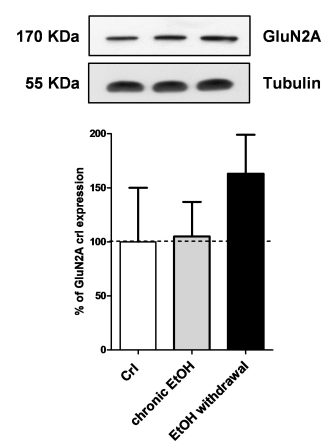

E

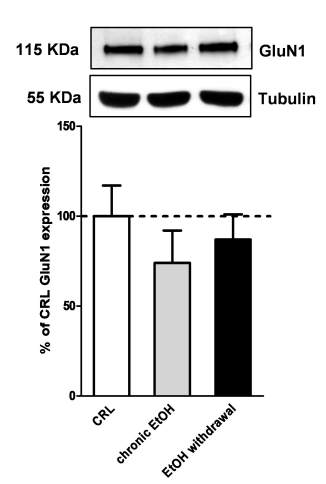

$\mathbf{F}$

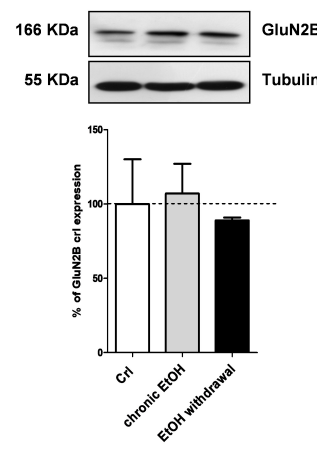

C

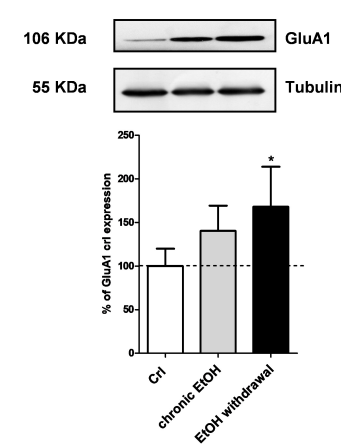

G
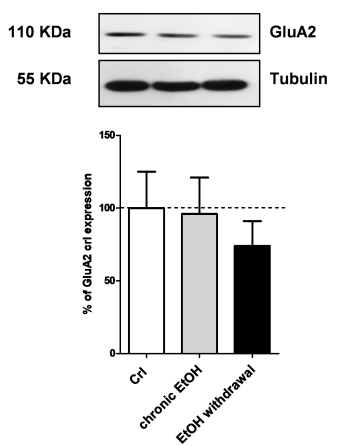

D

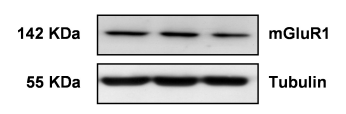

$55 \mathrm{KDa}$

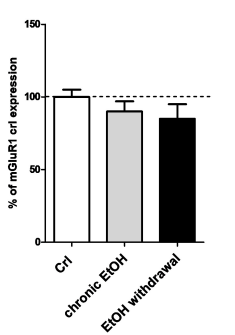

H

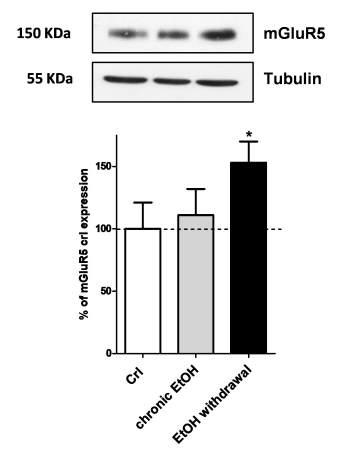

FIGURE 5 | Ethanol withdrawal increases the expression of the GluA1 AMPA subunit and of mGluR5 proteins in organotypic hippocampal slices. Experiments were conducted as described in Figure 1A. At the end of the experiments hippocampal slices were lysed and processed for Western blot. Top: representative Western blots using antibodies against the post-synaptic proteins PSD95, GluN1, GluN2A, GluN2B, GluA1, GluA2, mGluR1, and mGluR5 (A-H). The numbers on the left indicate the position of the molecular mass markers $(\mathrm{kDa})$. Tubulin was used as loading control. Bottom: quantitative analysis of immunoreactive bands, showing that ethanol withdrawal increases the expression of the AMPA subunit GluA1 and of metabotropic glutamate receptors mGluR5. Data are expressed as percentage of control. Bars represent the mean \pm SEM of at least three experiments. ${ }^{*} P<0.05$ vs. CRL (ANOVA + Dunnet's test).

glutamatergic post-synaptic transmission may be enhanced both during $\mathrm{EtOH}$ dependence and following withdrawal. Several reports have demonstrated that glutamatergic transmission is involved in $\mathrm{EtOH}$ dependence and withdrawal (Läck et al., 2007; Marty and Spigelman, 2012; Gerace et al., 2016). For example, elevated levels of glutamate have been measured in the medial prefrontal cortex of alcoholic patients and in nucleus accumbens of alcohol-dependent rats during acute withdrawal (Hermann et al., 2012; Pati et al., 2016). Our findings strongly suggest that incubation with $\mathrm{EtOH}$ and/or its removal markedly impair the structure and function of the excitatory post-synaptic compartments. On the contrary, in immature organotypic hippocampal slices EtOH induced a decrease in sEPSC frequency and in the expression of specific presynaptic proteins, thus suggesting an impairment of the structure and function of the excitatory pre-synaptic compartments (Gerace et al., 2016).

Moreover, data obtained by Western blotting showing that GluA1 and metabotropic Glu5 receptor expression levels were significantly increased in slices following chronic EtOH incubation and its withdrawal from the medium. On the contrary, no changes in the expression of a number of presynaptic proteins (vGlut1, vGlut2, CB1, and synaptophysin) nor of the scaffold protein PSD95, the NMDA subunits GluN1, GluN2A and GluN2B, the AMPA subunit GluA2 or mGlu1 receptors were observed. Despite NMDA receptor alterations have been described to contribute to neuronal excitation and neurotoxicity following EtOH withdrawal (Butler et al., 2013), we failed to observe any significant change in the expression of its subunits GluN1, GluN2A, and GluN2B under our experimental conditions. Rather, our findings show that following chronic EtOH incubation and its withdrawal there is an increase in AMPA-mediated sEPSCs and in the expression of the GluA1 AMPA subunit and mGlu5 receptors (Figure 7), in agreement with reports demonstrating the implication of AMPA and mGlu5 receptors in various models of $\mathrm{EtOH}$ dependence and withdrawal. For example Varodayan et al. (2018) have shown that AMPA receptor-mediated excitatory transmission is enhanced in the medial prefrontal cortex of mice after chronic exposure to EtOH followed by 1 week of withdrawal. Similarly Li et al. (2017) have shown that the amplitude of AMPA EPSCs and GluA1 (S831) phosphorylation, that plays a crucial role in increasing AMPAR activity in various regions of the brain (Malinow, 2003), were increased in neurons of the lateral habenula after $24 \mathrm{~h}$ of withdrawal from chronic voluntary EtOH drinking. Consistent with these findings, AMPARs have been shown to play a key role in the regulation of reinforcement and reward processes of 
A

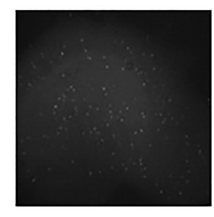

CRL

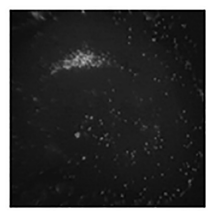

EtOH

withdrawa

$\stackrel{+}{N B Q X}$

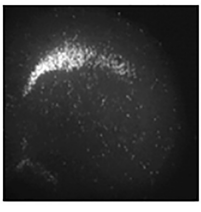

EtOH withdrawal

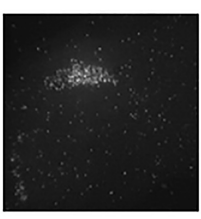

$\mathrm{EtOH}$

withdrawal

$\stackrel{+}{\stackrel{+}{M}}$
B

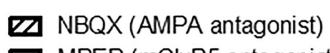

$\square \operatorname{MPEP}$ (mGluR5 antagonist)

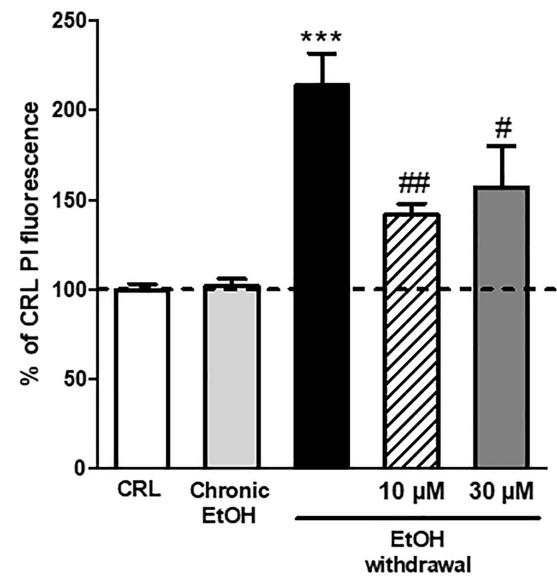

FIGURE 6 | AMPA and mGluR5 antagonists attenuate the neurotoxicity induced by ethanol withdrawal in organotypic hippocampal slices. (A): Hippocampal slices, photographed under fluorescence optics, displaying background levels of fluorescence under control conditions, an intense PI labeling in the CA1 subregion $24 \mathrm{~h}$ after ethanol withdrawal, and a reduction of CA1 PI fluorescence when incubated with $10 \mu \mathrm{M} \mathrm{NBQX}$ and $30 \mu \mathrm{M}$ of MPEP during the $24 \mathrm{~h}$ withdrawal period. (B): Quantitative analysis of CA1 region expressed as percentage of CRL PI fluorescence. Incubation with the AMPA antagonist NBQX and with the selective mGluR5 antagonist MPEP significantly attenuated ethanol-withdrawal-induced injury. Values represent the mean $\pm \mathrm{SEM}$ of at least five experiments. ${ }^{* * *} P<0.001$ vs. CRL and $\#<0.05$ vs. ethanol withdrawal alone and $\#^{\#}<0.01$ vs. ethanol withdrawal alone (ANOVA + Tukey's $w$ test).

Control
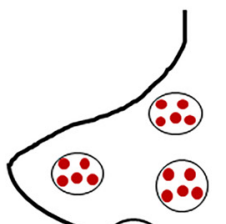

6

8.8

(8.)

$\because$

-

$\bullet$

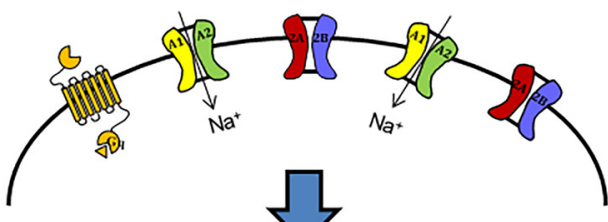

Physiological condition
EtOH withdrawal

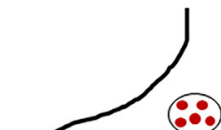

?

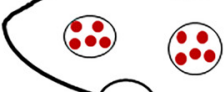

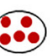

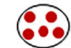

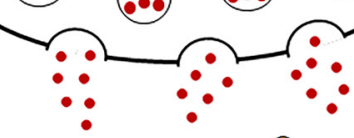

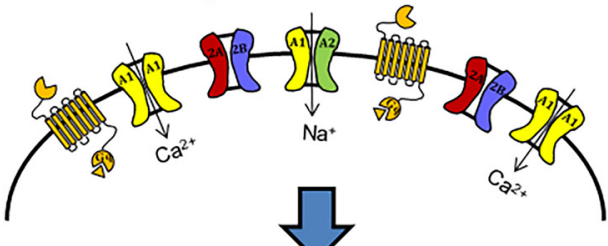

Pathological condition
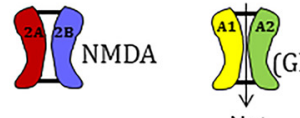

AMPA GluA1/GluA2)

$\mathrm{Na}^{+}$
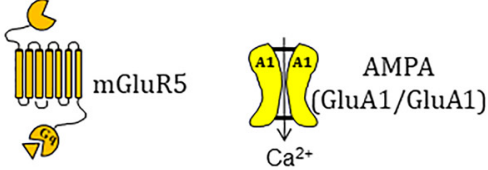

FIGURE 7 | Hypothetical model to explain mechanisms underlying ethanol withdrawal toxicity. Ethanol withdrawal potentiates the excitatory synaptic transmission that lead to CA1 injury of hippocampus in vitro. These alterations are probability due to the increase of the expression of the glutamate receptors AMPA (specifically the GluA1 AMPA subunit) and mGluR5. These evidences suggest that after ethanol withdrawal there is a shift in AMPAR subunit composition with the insertion of calcium permeable AMPA receptors in organotypic hippocampal slices. 
EtOH (Stuber et al., 2008; Wang et al., 2012), presumably via an EtOH-dependent increase in the surface concentration of GluA1 subunits in the striatum. As for mGlu5 receptors, a significant increase in its gene expression was observed in the amygdala and in the nucleus accumbens shell of EtOH-withdrawn rats (Kumar et al., 2016; Lee et al., 2018). Moreover, preclinical research suggests an important role of this receptor subtype in EtOH drinking (Cozzoli et al., 2009, 2012; Besheer et al., 2010; Sinclair et al., 2012) and in memory formation, which appears to be responsible for the chronic relapsing nature of alcohol abuse (Obara et al., 2009). In fact, mGlu5 has been implicated in cognition (e.g., memory and learning), novelty seeking behavior, and compulsivity, all factors that may be important in the susceptibility to drug addiction (Leurquin-Sterk et al., 2018).

We observed that both the AMPA competitive antagonist NBQX and the selective mGluR5 non-competitive antagonist MPEP were able to significantly attenuate EtOH withdrawalinduced neurotoxicity in the CA1 hippocampal sub-region, confirming the central role of AMPA channels and mGlu5 receptors in EtOH withdrawal hippocampal injury. The present findings support the results from reports showing that AMPA and mGlu5 receptor antagonists are involved in EtOH-withdrawalrelated behaviors. For example, pharmacological inhibition of AMPA receptors reduces EtOH consumption in rats (Li et al., 2017) and the systemic administration of mGlu5 antagonists attenuate cue-evoked reinstatement of ethanol-seeking behavior in rodents (Bäckström and Hyytiä, 2004; Adams et al., 2008; Hodge et al., 2008; Sinclair et al., 2012). Similarly, studies with mGlu5 negative allosteric modulators performed in animal models of addiction have shown a reduction in the selfadministration of cocaine, ethanol, and nicotine (Mihov and Hasler, 2016). Finally, our Western blot results show an increase in the expression of GluA1 but not GluA2 AMPA subunits in hippocampal slices following EtOH withdrawal, suggesting a shift in AMPA receptor subunit composition, from GluA2-containing to GluA2-lacking calcium-permeable channels that could explain the CA1 injury observed in our slices following EtOH-withdrawal. An increase in GluA2-lacking calcium-permeable AMPA channels has been shown to promote

\section{REFERENCES}

Adams, C. L., Cowen, M. S., Short, J. L., and Lawrence, A. J. (2008). Combined antagonism of glutamate mGlu5 and adenosine A2A receptors interact to regulate alcohol-seeking in rats. Int. J. Neuropsychopharmacol. 11, 229-241. doi: 10.1017/S1461145707007845

Anzai, T., Tsuzuki, K., Yamada, N., Hayashi, T., Iwakuma, M., Inada, K., et al. (2003). Overexpression of Ca2+-permeable AMPA receptor promotes delayed cell death of hippocampal CA1 neurons following transient forebrain ischemia. Neurosci. Res. 46, 41-51. doi: 10.1016/S0168-0102(03)00023-3

Bäckström, P., and Hyytiä, P. (2004). Ionotropic glutamate receptor antagonists modulate cue-induced reinstatement of ethanol-seeking behavior. Alcohol. Clin. Exp. Res. 28, 558-565. doi: 10.1097/01.ALC.0000122101.13 164.21

Besheer, J., Grondin, J. J., Cannady, R., Sharko, A. C., Faccidomo, S., and Hodge, C. W. (2010). Metabotropic glutamate receptor 5 activity in the nucleus accumbens is required for the maintenance of ethanol self-administration in a rat genetic model of high alcohol intake. Biol. Psychiatry 67, 812-822. doi: 10.1016/j.biopsych.2009.09.016 cell death of hippocampal CA1 pyramidal neurons in models of toxicity (Anzai et al., 2003; Gerace et al., 2014) and in pathological conditions including global ischemia (Pellegrini-Giampietro et al., 1997) and amyotrophic lateral sclerosis (Hideyama and Kwak, 2011). Calcium-permeable AMPA receptors have also been demonstrated to play an important role in addiction models (Mameli et al., 2011; Pascoli et al., 2014): for example, it was recently shown that cocaine-induced potentiation of VTA synapses is mediated by the insertion of calcium permeable AMPA receptors to the synaptic membranes (Mills et al., 2017).

\section{CONCLUSION}

Our data suggest that chronic EtOH treatment promotes abnormal synaptic transmission that may lead to CA1 pyramidal cell death after EtOH withdrawal through glutamate receptors and increased excitotoxicity. In addition, we propose AMPA and mGlu5 receptors as two candidate targets for new therapeutic interventions for alcohol addiction.

\section{AUTHOR CONTRIBUTIONS}

EG designed the research studies, conducted the experiments, acquired and analyzed the data, and wrote the manuscript. EL and DB conducted the experiments and acquired and analyzed the data. FM designed the research studies and edited the manuscript. GM designed the research studies, provided reagents, and edited the manuscript. DP-G designed the research studies, provided reagents, and wrote the manuscript.

\section{FUNDING}

This work was supported by grants from the Ente Cassa di Risparmio di Firenze, and from the University of Florence. EG is the recipient of a Zardi-Gori fellowship.

Butler, T. R., Berry, J. N., Sharrett-Field, L. J., Pauly, J. R., and Prendergast, M. A. (2013). Long-term ethanol and corticosterone co-exposure sensitize the hippocampal cal region pyramidal cells to insult during ethanol withdrawal in an NMDA GluN2B subunit-dependent manner. Alcohol. Clin. Exp. Res. 37, 2066-2073. doi: 10.1111/acer.12195

Charlet, K., Beck, A., and Heinz, A. (2013). The dopamine system in mediating alcohol effects in humans. Curr. Top. Behav. Neurosci. 13, 461-488. doi: 10. 1007/7854_2011_130

Cozzoli, D. K., Courson, J., Caruana, A. L., Miller, B. W., Greentree, D. I., Thompson, A. B., et al. (2012). Nucleus accumbens mGluR5associated signaling regulates binge alcohol drinking under drinking-in-thedark procedures. Alcohol. Clin. Exp. Res. 36, 1623-1633. doi: 10.1111/j.15300277.2012.01776.x

Cozzoli, D. K., Goulding, S. P., Zhang, P. W., Xiao, B., Hu, J. H., Ary, A. W., et al. (2009). Binge drinking upregulates accumbens mGluR5-Homer2-PI3K signaling: functional implications for alcoholism. J. Neurosci. 29, 8655-8668. doi: 10.1523/JNEUROSCI.5900-08.2009

Gass, J. T., and Olive, M. F. (2008). Glutamatergic substrates of drug addiction and alcoholism. Biochem. Pharmacol. 75, 218-265. doi: 10.1016/j.bcp.2007.06.039 
Gerace, E., Landucci, E., Scartabelli, T., Moroni, F., Chiarugi, A., and PellegriniGiampietro, D. E. (2015). Interplay between histone acetylation/deacetylation and poly(ADP-ribosyl)ation in the development of ischemic tolerance in vitro. Neuropharmacology 92, 125-134. doi: 10.1016/j.neuropharm.2015.01.008

Gerace, E., Landucci, E., Scartabelli, T., Moroni, F., and Pellegrini-Giampietro, D. E. (2012a). Rat hippocampal slice culture models for the evaluation of neuroprotective agents. Methods Mol. Biol. 846, 343-354. doi: 10.1007/978-161779-536-7_29

Gerace, E., Scartabelli, T., Formentini, L., Landucci, E., Moroni, F., Chiarugi, A., et al. (2012b). Mild activation of poly(ADP-ribose) polymerase (PARP) is neuroprotective in rat hippocampal slice models of ischemic tolerance. Eur. J. Neurosci. 36, 1993-2005. doi: 10.1111/j.1460-9568.2012.08116.x

Gerace, E., Landucci, E., Totti, A., Bani, D., Guasti, D., Baronti, R., et al. (2016). Ethanol toxicity during brain development: alterations of excitatory synaptic transmission in immature organotypic hippocampal slice cultures. Alcohol. Clin. Exp. Res. 40, 706-716. doi: 10.1111/acer.13006

Gerace, E., Masi, A., Resta, F., Felici, R., Landucci, E., Mello, T., et al. (2014). PARP-1 activation causes neuronal death in the hippocampal CA1 region by increasing the expression of $\mathrm{Ca}(2+)$-permeable AMPA receptors. Neurobiol. Dis. 70, 43-52. doi: 10.1016/j.nbd.2014.05.023

Harris, B. R., Gibson, D. A., Prendergast, M. A., Blanchard, J. A., Holley, R. C., Hart, S. R., et al. (2003). The neurotoxicity induced by ethanol withdrawal in mature organotypic hippocampal slices might involve cross-talk between metabotropic glutamate type 5 receptors and N-methyl-D-aspartate receptors. Alcohol. Clin. Exp. Res. 27, 1724-1735. doi: 10.1097/01.ALC.0000093601.33119.E3

Hermann, D., Weber-Fahr, W., Sartorius, A., Hoerst, M., Frischknecht, U., Tunc-Skarka, N., et al. (2012). Translational magnetic resonance spectroscopy reveals excessive central glutamate levels during alcohol withdrawal in humans and rats. Biol. Psychiatry 71, 1015-1021. doi: 10.1016/j.biopsych.2011. 07.034

Hideyama, T., and Kwak, S. (2011). When does ALS start? ADAR2-GluA2 hypothesis for the etiology of sporadic ALS. Front. Mol. Neurosci. 4:33. doi: 10.3389/fnmol.2011.00033

Hodge, C. W., Miles, M. F., Sharko, A. C., Stevenson, R. A., Hillmann, J. R., Lepoutre, V., et al. (2008). The mGluR5 antagonist MPEP selectively inhibits the onset and maintenance of ethanol self-administration in C57BL/6J mice. Psychopharmacology 183, 429-438. doi: 10.1007/s00213-005-0217-y

Kalivas, P. W., and Volkow, N. D. (2005). The neural basis of addiction: a pathology of motivation and choice. Am. J. Psychiatry 162, 1403-1413. doi: 10.1176/appi. ajp.162.8.1403

Koob, G. F. (2013). Addiction is a reward deficit and stress surfeit disorder. Front. Psychiatry 4:72. doi: 10.3389/fpsyt.2013.00072

Koob, G. F., and Le Moal, M. (2008). Addiction and the brain antireward system. Annu. Rev. Psychol. 59, 29-53. doi: 10.1146/annurev.psych.59.103006.09 3548

Krupitsky, E. M., Rudenko, A. A., Burakov, A. M., Slavina, T. Y., Grinenko, A. A., Pittman, B., et al. (2007). Antiglutamatergic strategies for ethanol detoxification: comparison with placebo and diazepam. Alcohol. Clin. Exp. Res. 31, 604-611. doi: 10.1111/j.1530-0277.2007.00344.X

Kumar, J., Hapidin, H., Get Bee, Y. T., and Ismail, Z. (2016). The effects of acute ethanol administration on ethanol withdrawal-induced anxiety-like syndrome in rats: a biochemical study. Alcohol 50, 9-17. doi: 10.1016/j.alcohol.2015.10.001

Läck, A. K., Diaz, M. R., Chappell, A., DuBois, D. W., and McCool, B. A. (2007). Chronic ethanol and withdrawal differentially modulate pre- and postsynaptic function at glutamatergic synapses in rat basolateral amygdala. J. Neurophysiol. 98, 3185-3196. doi: 10.1152/jn.00189.2007

Landucci, E., Filippi, L., Gerace, E., Catarzi, S., Guerrini, R., and PellegriniGiampietro, D. E. (2018). Neuroprotective effects of topiramate and memantine in combination with hypothermia in hypoxic-ischemic brain injury in vitro and in vivo. Neurosci. Lett. 6, 103-107. doi: 10.1016/j.neulet.2018.01.023

Landucci, E., Lattanzi, R., Gerace, E., Scartabelli, T., Balboni, G., Negri, L., et al. (2016). Prokineticins are neuroprotective in models of cerebral ischemia and ischemic tolerance in vitro. Neuropharmacology 108, 39-48. doi: 10.1016/j. neuropharm.2016.04.043

Lee, K. M., Coelho, M. A., Class, M. A., and Szumlinski, K. K. (2018). mGlu5dependent modulation of anxiety during early withdrawal from binge-drinking in adult and adolescent male mice. Drug Alcohol Depend 184, 1-11. doi: 10. 1016/j.drugalcdep.2017.10.031
LeMarquand, D., Pihl, R. O., and Benkelfat, C. (1994). Serotonin and alcohol intake, abuse, and dependence: findings of animal studies. Biol. Psychiatry 36, 395-421. doi: 10.1016/0006-3223(94)91215-7

Leurquin-Sterk, G., Ceccarini, J., Crunelle, C. L., Weerasekera, A., de Laat, B., Himmelreich, U., et al. (2018). Cerebral dopaminergic and glutamatergic transmission relate to different subjective responses of acute alcohol intake: an in vivo multimodal imaging study. Addict. Biol. 23, 931-944. doi: 10.1111/adb. 12542

Li, J., Kang, S., Fu, R., Wu, L., Wu, W., Liu, H., et al. (2017). Inhibition of AMPA receptor and CaMKII activity in the lateral habenula reduces depressive-like behavior and alcohol intake in rats. Neuropharmacology 126, 108-120. doi: 10.1016/j.neuropharm.2017.08.035

Malinow, R. (2003). AMPA receptor trafficking and long-term potentiation. Philos. Trans. R. Soc. Lond. B Biol. Sci. 358, 707-714. doi: 10.1098/rstb.2002. 1233

Mameli, M., Bellone, C., Brown, M. T., and Lüscher, C. (2011). Cocaine inverts rules for synaptic plasticity of glutamate transmission in the ventral tegmental area. Nat. Neurosci. 14, 414-416. doi: 10.1038/nn.2763

Marty, V. N., and Spigelman, I. (2012). Long-lasting alterations in membrane properties, $\mathrm{k}(+)$ currents, and glutamatergic synaptic currents of nucleus accumbens medium spiny neurons in a rat model of alcohol dependence. Front. Neurosci. 8:86. doi: 10.3389/fnins.2012.00086

Mihov, Y., and Hasler, G. (2016). Negative allosteric modulators of metabotropic glutamate receptors subtype 5 in addiction: a therapeutic window. Int. J. Neuropsychopharmacol. 19:yw002. doi: 10.1093/ijnp/pyw002

Mills, F., Globa, A. K., Liu, S., Cowan, C. M., Mobasser, M., Phillips, A. G., et al. (2017). Cadherins mediate cocaine-induced synaptic plasticity and behavioral conditioning. Nat. Neurosci. 20, 540-549. doi: 10.1038/nn. 4503

Nagy, J., Kolok, S., Boros, A., and Dezso, P. (2005). Role of altered structure and function of NMDA receptors in development of alcohol dependence. Curr. Neuropharmacol. 3, 281-297. doi: 10.2174/15701590577432 2499

Nam, H. W., McIver, S. R., Hinton, D. J., Thakkar, M. M., Sari, Y., Parkinson, F. E., et al. (2012). Adenosine and glutamate signaling in neuron-glial interactions: implications in alcoholism and sleep disorders. Alcohol. Clin. Exp. Res. 36, 1117-1125. doi: 10.1111/j.1530-0277.2011.01722.x

Obara, I., Bell, R. L., Goulding, S. P., Reyes, C. M., Larson, L. A., Ary, A. W., et al. (2009). Differential effects of chronic ethanol consumption and withdrawal on homer/glutamate receptor expression in subregions of the accumbens and amygdala of P rats. Alcohol. Clin. Exp. Res. 33, 1924-1934. doi: 10.1111/j.15300277.2009.01030.x

Pascoli, V., Terrier, J., Espallergues, J., Valjent, E., O’Connor, E. C., and Lücher, C. (2014) Contrasting forms of cocaine-evoked plasticity control components of relapse. Nature 509, 459-464. doi: 10.1038/nature13257

Pati, D., Kelly, K., Stennett, B., Frazier, C. J., and Knackstedt, L. A. (2016). Alcohol consumption increases basal extracellular glutamate in the nucleus accumbens core of sprague-dawley rats without increasing spontaneous glutamate release. Eur. J. Neurosci. 44, 1896-1905. doi: 10.1111/ejn. 13284

Pellegrini-Giampietro, D. E., Gorter, J. A., Bennett, M. V., and Zukin, R. S. (1997). The GluR2 (GluR-B) hypothesis: $\mathrm{Ca}(2+)$-permeable AMPA receptors in neurological disorders. Trends Neurosci. 20, 464-470. doi: 10.1016/S01662236(97)01100-4

Pellegrini-Giampietro, D. E., Peruginelli, F., Meli, E., Cozzi, A., Albani Torregrossa, S., Pellicciari, R., et al. (1999). Protection with metabotropic glutamate 1 receptor antagonists in models of ischemic neuronal death: timecourse and mechanisms. Neuropharmacology 38, 1607-1619. doi: 10.1016/ S0028-3908(99)00097-0

Prendergast, M. A., Harris, B. R., Mayer, S., and Littleton, J. M. (2000). Chronic, but not acute, nicotine exposure attenuates ethanol withdrawal-induced hippocampal damage in vitro. Alcohol. Clin. Exp. Res. 24, 1583-1592. doi: 10.1111/j.1530-0277.2000.tb04578.x

Self, R. L., Smith, K. J., Mulholland, P. J., and Prendergast, M. A. (2005). Ethanol exposure and withdrawal sensitizes the rat hippocampal CA1 pyramidal cell region to beta-amyloid (25-35)-induced cytotoxicity: NMDA receptor involvement. Alcohol. Clin. Exp. Res. 29, 2063-2069. doi: 10.1097/01.alc. 0000187591.82039.b2 
Sinclair, C. M., Cleva, R. M., Hood, L. E., Olive, M. F., and Gass, J. T. (2012). mGluR5 receptors in the basolateral amygdala and nucleus accumbens regulate cue-induced reinstatement of ethanol-seeking behavior. Pharmacol. Biochem. Behav. 101, 329-335. doi: 10.1016/j.pbb.2012.01.014

Stuber, G. D., Klanker, M., de Ridder, B., Bowers, M. S., Joosten, R. N., Feenstra, M. G., et al. (2008). Reward-predictive cues enhance excitatory synaptic strength onto midbrain dopamine neurons. Science 321, 1690-1692. doi: 10. $1126 /$ science. 1160873

Tabakoff, B., and Hoffman, P. L. (2013). The neurobiology of alcohol consumption and alcoholism: an integrative history. Pharmacol. Biochem. Behav. 113, 20-37. doi: 10.1016/j.pbb.2013.10.009

Tsai, G., and Coyle, J. T. (1998). The role of glutamatergic neurotransmission in the pathophysiology of alcoholism. Annu. Rev. Med. 49, 173-184. doi: 10.1146/ annurev.med.49.1.173

Varodayan, F. P., Sidhu, H., Kreifeldt, M., Roberto, M., and Contet, C. (2018). Morphological and functional evidence of increased excitatory signaling in the prelimbic cortex during ethanol withdrawal. Neuropharmacology 133, 470-480. doi: 10.1016/j.neuropharm.2018.02.014
Wang, J., Ben Hamida, S., Darcq, E., Zhu, W., Gibb, S. L., Lanfranco, M. F., et al. (2012). Ethanol-mediated facilitation of AMPA receptor function in the dorsomedial striatum: implications for alcohol drinking behavior. J. Neurosci. 32, 15124-15132. doi: 10.1523/JNEUROSCI.2783-12.2012

World Health Organization [WHO] (2014). Global Status Report on Alcohol and Health 2014. Geneva: World Health Organization.

Conflict of Interest Statement: The authors declare that the research was conducted in the absence of any commercial or financial relationships that could be construed as a potential conflict of interest.

Copyright (c) 2019 Gerace, Landucci, Bani, Moroni, Mannaioni and PellegriniGiampietro. This is an open-access article distributed under the terms of the Creative Commons Attribution License (CC BY). The use, distribution or reproduction in other forums is permitted, provided the original author(s) and the copyright owner(s) are credited and that the original publication in this journal is cited, in accordance with accepted academic practice. No use, distribution or reproduction is permitted which does not comply with these terms. 